\title{
ORIGINAL
}

\section{ESTUDIO cYcEVA: CASOS Y CONTROLES PARA LA ESTIMACIÓN DE LA EFECTIVIDAD DE LA VACUNA ANTIGRIPAL EN ESPAÑA, 2008-2013 (*)}

Silvia Jiménez-Jorge (1,2), Salvador de Mateo Ontañón (1,2), Camelia Savulescu (1), Concepción Delgado-Sanz $(1,2)$, Francisco Pozo Sánchez (3), Manuel García-Cenoz (4,2), Jesús Castilla Catalán (4,2), Carolina Rodríguez Gay (5), Tomás Vega Alonso (5), Carmen Quiñones Rubio (6), Eva Martínez Ochoa (6), Juana María Vanrell Berga (7), Jaume Giménez Durán (7), Daniel Castrillejo Pérez (8), Jone Miren Altzíbar Arotzena (9,2), Fernando González Carril (10), Julián Mauro Ramos Aceitero (11), María del Carmen Serrano Martin (11), Ana Martínez i Mateo (12,2), Nuria Torner Gràcia (12,2), Esteban Pérez Morilla (13), Virtudes Gallardo García (13) y Amparo Larrauri Cámara $(1,2)$.

(1) Centro Nacional de Epidemiología. Instituto de Salud Carlos III. Madrid.

(2) Ciber Epidemiología y Salud Pública (CIBERESP).

(3) Centro Nacional de Microbiología. Instituto de Salud Carlos III. Madrid. España.

(4) Instituto de Salud Pública de Navarra.

(5) Dirección General de Salud Pública. Consejería de Sanidad de Castilla y León.

(6) Servicio de Epidemiología y Prevención Sanitaria. Dirección General de Salud Pública y Consumo de La Rioja.

(7) Servicio de Epidemiología. Dirección General de Salut Pública. Baleares.

(8) Servicio de Epidemiología. Dirección General de Sanidad y Consumo. Consejería de Bienestar Social y Sanidad. Ciudad Autónoma de Melilla.

(9) Dirección de Salud Pública de Gipuzkoa. País Vasco.

(10) Servicio de Salud Pública. Departamento de Salud. Gobierno del País Vasco.

(11) Dirección General de Salud Pública. Servicio Extremeño de Salud. Junta deExtremadura.

(12) Servicio de Vigilancia Epidemiológica. Dirección General de Salud Pública. Departament de Salut. Generalitat Catalunya. Cataluña.

(13) Servicio de Epidemiología y Salud Laboral. Secretaría General de Salud Pública y Participación. Consejería de Salud de Andalucía.

(*) Financiación: El estudio fue financiado por el European Centre for Disease Prevention and Control (ECDC tender OJ/2007/07/30-PROC/2007/015) a través de la Red para la vigilancia de la efectividad de la vacuna antigripal en Europa (IMOVE) y por el Instituto de Salud Carlos III [Programa de Investigación sobre la nueva Gripe AH1N1Influenza A(H1N1)pdm09 Programme (GR09/0017)]. Este proyecto recibió financiación adicional del Centers for Disease Control and Prevention de Estados Unidos, de la Oficina Europea de la Organización Mundial de la Salud y de Epiconcept, Francia. No existen conflictos de intereses.

\section{RESUMEN}

Fundamentos: Desde 2008-09 la efectividad de la vacuna (EV) antigripal en España se estima con el estudio de casos y controles para la evaluación de la EV antigripal (cycEVA), componente español de la red europea (Influenza-Monitoring Vaccine Effectiveness (I-MOVE). El objetivo es describir la evolución del estudio cycEVA durante las cinco temporadas del período 2008/09-2012/13.

Métodos: Se analizaron los siguientes indicadores: 1) participación de los médicos/pediatras centinela (MP); 2) población y periodo de estudio, 3 ) calidad de los datos y 4) difusión de los resultados mediantes publicaciones. Se calculó el porcentaje anual de cambio constante de los indicadores analizándose su tendencia mediante el test de Cochran-Armitage.

Resultados: El número de MP participantes aumentó de 164 en 2008-09 hasta 246 en ediciones posteriores. El porcentaje de médicos que reclutaron al menos un paciente experimentó un cambio anual significativo (PCA) del $15,33 \%$. El porcentaje de pacientes reclutados incluidos en el análisis aumentó del $77 \%$ en $2008-09$ a más del $95 \%$ en las siguientes ediciones $(\mathrm{PCA}=5,91 \%)$. El porcentaje de casos y controles participantes en cycEVA $(\mathrm{PCA}=5,91 \%)$. El porcentaje de casos y controles participantes en cycEVA
sobre el total de pacientes que contribuyeron al estudio europeo I-MOVE osciló entre el $23 \%$ en la edición piloto y $30 \%$ en la temporada 2011-12. Los resultados finales se difundieron en revistas científicas con un factor de impacto situado en el cuartil 2 y en 2010-11 y 2011-12 se publicaron resultados preliminares en revistas con un factor de impacto situado en el cuartil 1 (97 citas).

Conclusiones: La experiencia del estudio cycEVA se reflejó en una mejora en la oportunidad e impacto de sus resultados, cruciales para orientar las recomendaciones anuales de vacunación antigripal

Palabras clave: Gripe humana. Vigilancia centinela. Vacuna antigripal. Efectividad vacunal. Estudios de casos y controles.

Correspondencia

Silvia Jiménez Jorge

Área de Vigilancia de la Salud Pública, Centro Nacional de Epidemiología Instituto de Salud Carlos III

C/ Monforte de Lemos 5, Pabellón 12

28029 Madrid

sjimenezj@isciii.es

\section{ABSTRACT \\ cycEVA Study: Case Control Study Measuring Influenza Vaccine Effectiveness in Spain, 2008-2013}

Background: In Spain, influenza vaccine effectiveness (EV) is estimated since 2008-09 season through the cycEVA case-control study, the Spanish component of the European I-MOVE (Monitoring Influenza Vaccine Effectiveness in the EU/EEA) network. We aimed at describing cycEVA performance in its five consolidated editions 2008/09-2012/13.

Methods: During the study period the following indicators were analysed: 1) the participation of sentinel general practitioners and pediatricians (MP), 2) the population studied and the study period, 3) the data quality and 4) the dissemination of the cycEVA results. Trend analysis of the indicators was done using the Cochran-Armitage test to compute the Annual Percentage Change (PCA).

Results: The number of participating MP increased from 164 in 2008-09 to 246 in the following editions. The percentage of MP recruiting at least one patient increased significantly annually $(\mathrm{PCA}=15.33 \%)$. The percentage of recruited patients included into the analysis increased $(\mathrm{PCA}=5.91 \%)$ from $77 \%$ in 2008-09 to more than $95 \%$ in the following editions. The percentage of cycEVA patients contributing to the I-MOVE study ranged between $23 \%$ and $30 \%$ in the pilot and 2011-12 editions respectively.. Final results were disseminated in quartile 2 peer-reviewed journals and 2010-11 and 2011-12 preliminary EV estimates were published in quartile 1 journals. cycEVA publications received 97 citations.

Conclusion: cycEVA study achieved more quality information, timely EV estimates and a higher impact of the results.

Keyword: Human influenza. Sentinel Surveillance. Influenza vaccine. Vaccine effectiveness. Case-control studies. 


\section{INTRODUCCIÓN}

La vacunación antigripal anual de los grupos de riesgo se considera la mejor medida de prevención disponible actualmente para reducir el impacto de las epidemias estacionales o pandemias de gripe ${ }^{1}$. Esta vacuna se caracteriza por ser la única que se reformula cada año en respuesta a los cambios antigénicos constantes en los virus gripales $^{2}$.

Su efectividad varía de año en año en función, entre otros, del grado de concordancia entre las cepas vacunales y las circulantes, la edad de los sujetos vacunados, su estado inmunológico así como la presencia de memoria inmunológica. Por ello, la evaluación anual del programa de vacunación antigripal se ha convertido en prioritaria en los países de nuestro entorno, que recomiendan esta intervención de salud pública tanto a personas con alto riesgo de sufrir complicaciones por gripe como a las que los atienden, especialmente los profesionales sanita$\operatorname{rios}^{3,4}$.

La necesidad de la monitorización de la vacuna antigripal en Europa impulsó que el Centro Europeo para la Prevención y Control de Enfermedades (ECDC por sus siglas en inglés) financiara la creación de la red europea Influenza-Monitoring Vaccine Effectiveness (I-MOVE) $)^{5-6}$ con el objetivo de desarrollar un sistema capaz de estimar anualmente y en tiempo real la efectividad de esta vacuna en los países de la Unión Europea (UE) y el Espacio Económico Europeo. Los países participantes en la red I-MOVE consensuaron un protocolo común $^{7}$ que incluye una toma sistemática de muestra respiratoria a los pacientes participantes, registro de información sobre posibles factores de confusión y el uso de la definición de caso de gripe de la $\mathrm{UE}^{8}$. A partir de la fase piloto 2008-09 se utilizó el diseño test-negativo de $\operatorname{casos}^{9-13}$, estimándose la efectividad vacunal (EV) antigripal frente a la infección de gripe confirmada por laboratorio. Los casos son los sujetos que consultan al médico centinela por síndrome gripal y con resultado virológico positivo para virus gripales. Los controles quienes tienen resultados negativos. La EV antigripal se estima mediante modelos de regresión logística ajustando por posibles factores de confusión.

Desde 2008 España ha participado en la red europea I-MOVE con el estudio cycEVA, desarrollado en el marco del Sistema centinela de vigilancia de gripe en España (ScVGE). Las redes centinela participantes en el estudio se comprometieron a cumplir el protocolo europeo del estudio con una recogida exhaustiva de información de los pacientes a través del cuestionario consensuado $^{7}$ que incluye información adicional a la habitual de vigilancia: fecha de vacunación y tipo de vacuna antigripal administra$\mathrm{da}$, antecedentes de vacunación antigripal previa, hábito tabáquico y variables relacionadas con la gravedad y la frecuentación al médico.

El objetivo de este trabajo es describir la evolución del estudio cycEVA, desde la temporada 2008-09 hasta su quinta edición consolidada 2012-13.

\section{MATERIAL Y MÉTODOS}

Los indicadores analizados en el período de estudio, 2008-2013 fueron:

1) La participación de las redes y médi$\cos /$ pediatras centinela (MP).

2) La población y el periodo de estudio.

3) La calidad de los datos.

4) La difusión de los resultados.

La participación de las redes se analizó mediante el porcentaje de redes que participaron en ediciones consecutivas del estudio. La participación de los MP se evaluó analizando la evolución del número de médicos 
por edición, la fidelidad de los mismos (porcentaje de MP que ya habían participado en la edición anterior) así como el porcentaje de médicos que reclutaron al menos un paciente por edición.

Para evaluar la calidad de los datos obtenidos se analizó el porcentaje de datos incompletos y la incorporación en sucesivas ediciones de nuevas variables de ajuste para controlar por posibles factores de confusión. También se analizó la evolución del porcentaje de pacientes incluidos en el estudio cycEVA y su contribución porcentual al estudio europeo I-MOVE con respecto al total de pacientes y casos confirmados incluidos en el análisis europeo. El número de países participantes en el estudio multicéntrico de casos y controles I-MOVE osciló entre 5 en la fase piloto 2008-09, 7 en las temporadas $2009-10$ y $2012-13$ y 8 en 2010-11 y 2011-126.

Para valorar la evolución de la difusión de los resultados se estudió la oportunidad de los mismos, el número de informes elaborados, las presentaciones a congresos y conferencias nacionales e internacionales y el número de publicaciones en revistas revisadas por pares por cada edición. El factor de impacto de los resultados de cycEVA se cuantificó con el cuartil de las revistas en las que se publicaron los resultados así como con el número y la fuente de las citas bibliográficas recibidas. Para el análisis de las citas de las publicaciones cycEVA se utilizaron las bases de datos multidisciplinares internacionales: Web of Science, Scopus y Google Académico.

Como medida resumen de la evolución de los indicadores en el periodo de estudio se utilizó el porcentaje de cambio anual constante (PCA), que describe la proporción de incremento o decremento de los indicadores por año. Igualmente se analizó la tendencia en la evolución de los indicadores mediante el test de Cochran-Armitage ${ }^{17}$, investigando las desviaciones de la linealidad.

\section{RESULTADOS}

En todas las temporadas del estudio cycEVA participaron 7 redes centinela pertenecientes al ScVGE distribuidas a lo largo del territorio nacional, exceptuando la 2010-11 en la que participaron 8 redes (figura 1$)$.

En la tabla 1 se resumen las principales características del estudio cycEVA durante el período 2008-2013.

El número de MP participantes aumentó de 164 en la edición piloto a 238 de media en las siguientes temporadas (tabla 1). El número de redes centinela que participaron con el $100 \%$ de los MP integrados en el ScVGE se duplicó de dos a cuatro desde las fases piloto y pandémica a las posteriores. Todo ello se tradujo en el aumento del porcentaje de población vigilada por las redes cycEVA con respecto a la población total cubierta por dichas redes en el ScVGE del $1,7 \%$ en la edición piloto al $2,1 \%$ en el periodo posterior 2009-13 $(\mathrm{P}<0,001)$.

El porcentaje de MP que habían participado en las temporadas anteriores fue del 85 $\%$. Asimismo el porcentaje de médicos que incluyeron al menos un paciente por edición aumentó del 41\% en la edición 2008-09 a situarse entre el 80 y el $85 \%$ en las siguientes ediciones (tabla 1) con un porcentaje de cambio anual (PCA) del 15,33\% (p tendencia $=0,0000 ; P$ desvío linealidad $=0,0000)$.

La población de estudio se amplió de los mayores de 64 años en la edición piloto a toda la población a partir de 2009 (tabla 1). Además, desde la primera temporada cycEVA post-pandémica en la que se recogió información sobre si el paciente pertenecía o no a los grupos susceptibles de ser vacunados, la EV antigripal también se pudo estimar para la población diana.

En las dos primeras ediciones cycEVA, el periodo del estudio coincidió con la tempo- 
Figura 1

Redes centinela participantes en el estudio cycEVA (número de temporadas de participación), 2008-2013

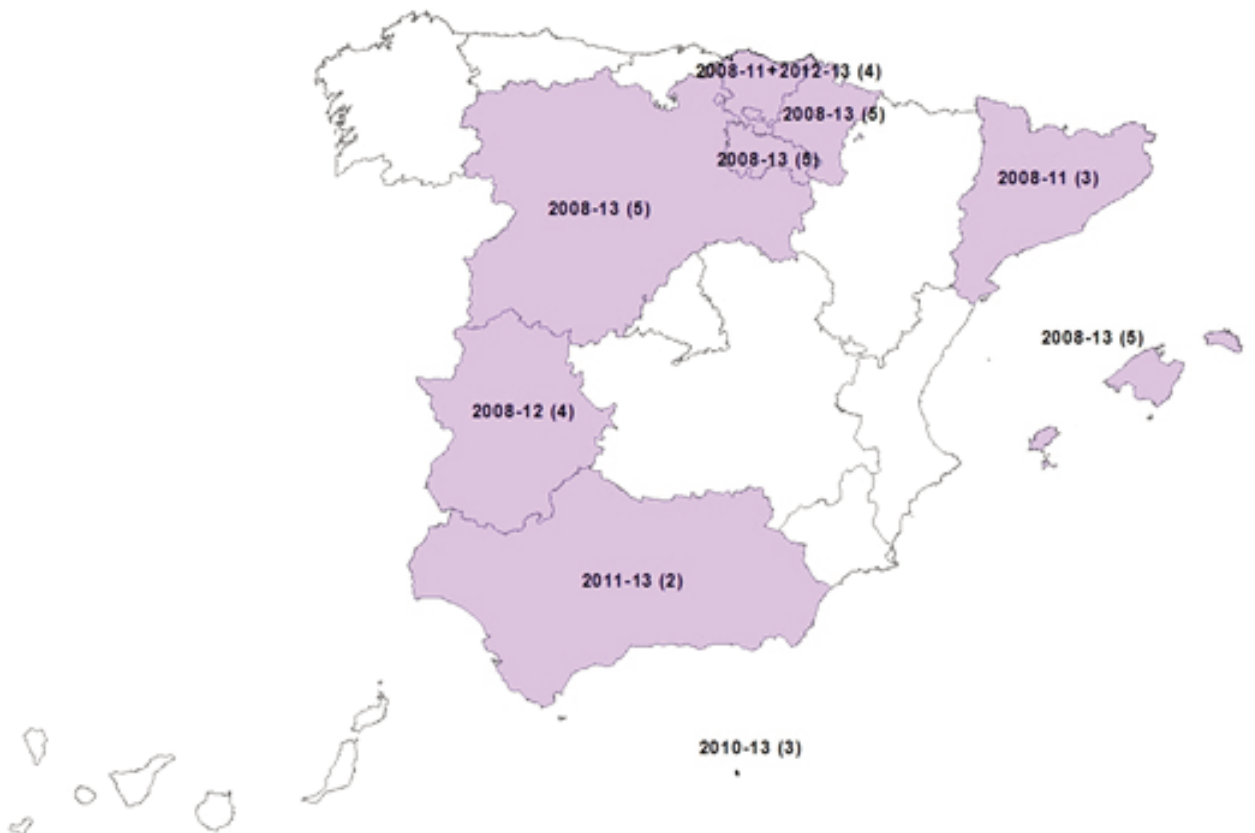

rada gripal, de la semana 40 de un año a la semana 20 del siguiente. Desde la temporada 2010-11 el periodo de estudio se restringió al periodo comprendido entre la semana en la que se inicia la onda epidémica de cada temporada, hasta que desaparece la circulación de los virus gripales en las redes participantes (semana en la que se identifica el último caso confirmado, seguida de dos semanas consecutivas sin casos de gripe confirmados).

Se observó una disminución en el porcentaje de datos incompletos en las variables de estudio, $0-20 \%$ en la edición piloto a $0-8 \%$ en las dos últimas temporadas 2011-12 y 2012-13 (tabla 1). Este hecho se tradujo en un aumento en el porcentaje de pacientes reclutados incluidos en el análisis $(77 \%$ en 2008-09 a más del 95\% en las siguientes ediciones), con un PCA del 5,91\% sin una tendencia significativa en los datos (para este análisis no se tuvo en cuenta la temporada pandémica por su carácter inusual).

El porcentaje de pacientes (casos y controles) cycEVA sobre el total de pacientes que contribuyeron al estudio europeo IMOVE osciló entre el 23\% y 30\% en las ediciones piloto y 2011-12, respectivamente, aumentando cada año un 9,54\% (sin tener en cuenta la temporada pandémica y última temporada consolidada) con una tendencia significativa $\left(\mathrm{X}^{2}{ }_{1}=24,117\right.$; $\mathrm{P}$ tendencia $=0,0000)$ que no difería de la linealidad $\left(\mathrm{X}_{3}^{2}=2.179\right.$; P desvío linealidad $\left.=0,1398\right)$. Asimismo, el PCA de casos confirmados cycEVA sobre el total de casos confirmados en I-MOVE fue del $13,94 \%$ (28\% en la temporada 2008-09 y 48\% en la edición 201112) con una tendencia significativa $\left(X^{2}{ }_{1}=\right.$ 24,117; P tendencia $=0.0000)$ sin diferir de la linealidad $\left(\mathrm{X}_{3}^{2}=2,467\right.$; $\mathrm{P}$ desvío linealidad $=0,1163$ ) en los datos (tabla 1 ). 
Tabla 1

Resumen de las características del estudio cycEVA, periodo 2008/09- 2012/13

\begin{tabular}{|c|c|c|c|c|c|}
\hline \multicolumn{6}{|c|}{ cycEVA } \\
\hline Características estudiadas & 2008-09 & $2009-10$ & $2010-11$ & 2011-12 & $2012-13$ \\
\hline $\begin{array}{l}\text { Médicos y pediatrasparticipantes } \\
/ \text { Redes }\left(\mathrm{n}^{\circ} / \mathrm{n}^{\circ}\right)\end{array}$ & $164 / 7$ & $235 / 7$ & $246 / 8$ & $231 / 7$ & $239 / 7$ \\
\hline $\begin{array}{l}\text { Población estudio } \\
\mathrm{n}^{\circ} \text { habitantes }\end{array}$ & $\begin{array}{l}\text { Mayores de } 64 \text { años } \\
46.461\end{array}$ & $\begin{array}{l}\text { Todas las edades } \\
310.005\end{array}$ & $\begin{array}{l}\text { Todas las edades } \\
400.416\end{array}$ & $\begin{array}{c}\text { Todas las edades } \\
453.158\end{array}$ & $\begin{array}{c}\text { Todas las edades } \\
304.647\end{array}$ \\
\hline $\begin{array}{l}\text { Periodo de estudio } \\
\text { (rango semanas) }\end{array}$ & $40 / 2008-20 / 2009$ & $40 / 2009-20 / 2010$ & $50 / 2010-12 / 2011$ & $52 / 2011-18 / 2012$ & $51 / 2012-17 / 2013$ \\
\hline $\begin{array}{l}\text { Nuevas variables incluidas } \\
\text { en el cuestionario* }\end{array}$ & & $\begin{array}{l}\text { Enfermedad renal crónica } \\
\text { Embarazo } \\
\text { Obesidad mórbida } \\
\text { (IMC } \geq 40 \text { ) } \\
\mathrm{N}^{\mathrm{o}} \text { de visitas en los } \\
\text { últimos } 12 \text { meses }\end{array}$ & $\begin{array}{l}\text { Enfermedad hepática crónica } \\
\text { Tratamiento AAS (en niños) } \\
\text { Pertenencia a un grupo } \\
\text { recomendado para } \\
\text { vacunación antigripal }\end{array}$ & Ninguna & Ninguna \\
\hline 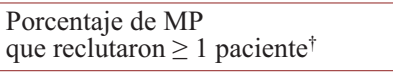 & $41 \%$ & $82 \%$ & $80 \%$ & $85 \%$ & $82 \%$ \\
\hline $\mathrm{N}^{o}$ pacientes reclutados ${ }^{\ddagger}$ & 103 & 1.545 & 1.376 & 1.471 & 1.459 \\
\hline $\begin{array}{l}\text { Porcentaje de datos incompletos } \\
\text { en las variables de estudio (rango) }\end{array}$ & $0-20 \%$ & $0-22 \%$ & $0-10 \%$ & $0-8 \%$ & $0-8 \%$ \\
\hline $\begin{array}{l}\text { Porcentaje de pacientes reclutados } \\
\text { incluidos en análisis }{ }^{\ddagger}\end{array}$ & $77 \%$ & $28 \%{ }^{\mathrm{a}}$ & $99 \%$ & & $96 \%$ \\
\hline $\begin{array}{l}\text { Porcentaje de pacientes cycEVA } \\
\text { que contribuyeron a I-MOVE } \\
(\% \text { casos confirmados })\end{array}$ & $23 \%(28 \%)$ & $10 \%(7 \%)^{\mathrm{a}}$ & $26 \%(34 \%)$ & $30 \%(48 \%)$ & $20 \%(23 \%)$ \\
\hline Difusión resultados & $\begin{array}{l}\text { Informe anual } \\
\text { Congresos: } \\
\text { Nac. (1), } \\
\text { Int. (1) } \\
\text { Publicación final }{ }^{16}\end{array}$ & $\begin{array}{l}\text { Informe anual } \\
\text { Congresos: Nac. (1), } \\
\text { Int. (2) } \\
\text { Publicación final }^{18}\end{array}$ & $\begin{array}{l}\text { Boletines semanales } \\
\text { Informe anual } \\
\text { Congresos: Nacionales } 2 \\
\text { Internacionales } 2 \\
\text { Publicaciones } \\
\text { preliminar y final } 1^{19,21}\end{array}$ & $\begin{array}{l}\text { Boletines semanales } \\
\text { Informe anual } \\
\text { Congresos: Nacionales } 2, \\
\text { Internacionales } 2 \\
\text { Publicaciones } \\
\text { preliminar y final }^{20,22}\end{array}$ & $\begin{array}{l}\text { Boletines semanales } \\
\text { Informe anual } \\
\text { Informe GIVE } \\
\text { Congresos: Nacionales } 2 \\
\text { Internacionales } 1 \\
\text { Publicación final }\end{array}$ \\
\hline
\end{tabular}

IMC: índice de masa corporal. AAS: ácido acetil salicílico. I-MOVE: Influenza Monitoring Vaccine Effectiveness in Europe GIVE: Global Influenza Vaccine Effectiveness collaboration.

*Además de las variables incluidas en el cuestionario desde la temporada piloto 2008-09: edad, sexo, síntomas clínicos, fechas de inicio de síntomas y toma de muestra, antecedentes de vacunación actual (fecha de vacunación y tipo de vacuna recibida), antecedentes de vacunación antigripal previa, resultados de laboratorio, hábito tabáquico, estado funcional (ayuda para andar y/o bañarse (variable no recogida a partir de la temporada 2010-11)), presencia de enfermedades crónicas (diabetes mellitus, enfermedades cardiovasculares, enfermedad pulmonar crónica, inmunodeficiencia congénita o adquirida) y $\mathrm{n}^{\circ}$ de hospitalizaciones por enfermedades crónicas en los 12 meses previos. ${ }^{\dagger}$ En el periodo de estudio de cada edición cycEVA. ${ }^{\ddagger}$ Pacientes reclutados en el periodo de estudio en los que la toma de muestra fue $<8$ días desde el inicio de los síntomas. a En la edición pandémica, el bajo porcentaje de pacientes reclutados incluidos en el análisis cycEVA y su contribución al estudio europeo I-MOVE se debe a que la vacuna pandémica no estuvo disponible hasta el pico epidémico y, por lo tanto, los pacientes incluidos en el estudio fueron aquéllos reclutados dos semanas después de que la vacuna estuviese disponible. 
Se incorporaron al cuestionario variables relacionadas con la presencia de enfermedades crónicas/factores de riesgo relacionados con gripe, información sobre la frecuentación médica de la población así como la pertenencia a un grupo recomendado de vacunación antigripal (tabla 1 ).

Los resultados obtenidos en cada edición cycEVA se difundieron nacional e internacionalmente a través de informes a las autoridades sanitarias, presentaciones a congresos y conferencias y publicaciones científicas con evaluación por pares. Las estimaciones preliminares y finales de EV de la edición cycEVA 2012-13, a través de su participación en la red europea I-MOVE, contribuyeron por primera vez en febrero y septiembre de 2013 a las recomendaciones de la Organización Mundial de la Salud (OMS) para la selección de cepas de virus de la gripe que se incluyeron en la vacuna antigripal de la temporada 2013-14 en el hemisferio norte y 2013 para el hemisferio sur mediante un informe elaborado por The Global Influenza Vaccine Effectiveness Collaboration (GIVE).
Para incentivar la participación de los médicos en el estudio, a partir de la temporada 2009-10 se editó un boletín semanal en el que se incluyó información sobre la evolución del estudio y el reclutamiento de los pacientes en cada edición cycEVA e IMOVE, junto con la situación de la actividad gripal en España.

En todas las ediciones los resultados del estudio se presentaron en al menos un congreso de ámbito nacional (dos en las últimas dos ediciones) y en uno de ámbito internacional (dos desde la temporada pandémica). En cuanto a la producción científica, los resultados finales de cada edición se publicaron en revistas con evaluación por pares cuyo factor de impacto se sitúa en el cuartil $2^{16,18-20}$ [Vaccine (2), BMC Public Health (1) y BMC Infectious Diseases (1) los resultados de la edición 2012-13 están actualmente en revisión editorial]. En las ediciones 2010-11 y 2011-12 se publicaron además estimaciones preliminares de EV antigripal en el pico de la onda epidémica ${ }^{21,22}$ en Eurosurveillance (revista del ECDC cuyo factor de impacto se sitúa en el cuartil 1).

\section{Figura 2}

Procedencia de las publicaciones que citaron artículos cycEVA, 2008-2013. Fuente: Web of Science, Scopus y Google Académico

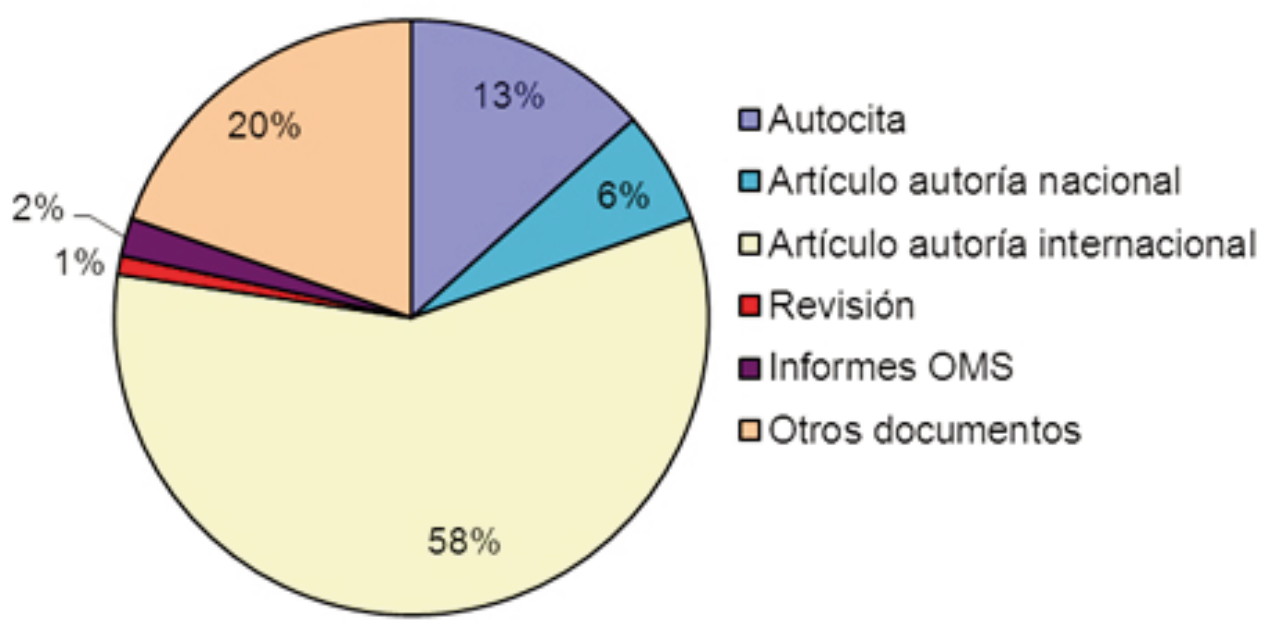


Con respecto al impacto de las publicaciones cycEVA, a 19 de marzo de 2014 sus artículos recibieron 97 citas, el 6\% fueron citas de autores nacionales, el 13\% autocitas, el $20 \%$ provenían de otros documentos (informes nacionales y revistas sin factor de impacto) y $58 \%$ fueron de autoría internacional. Cabe destacar la inclusión de dos de las seis publicaciones cycEVA en documentos internacionales: 1 revisión sistemática sobre la eficacia y efectividad de la vacuna antigripal ${ }^{23}$ y en 2 informes de la OMS, uno sobre la evaluación de vacunas antigripales pandémicas ${ }^{24}$ y otro sobre la actividad gripal en la temporada 2011-12 en el hemisferio norte ${ }^{25}$. El 79\% de las citas, excluyendo las autocitas, procedían de revistas cuyo factor de impacto se sitúa en el primer cuartil $(52,5 \%)$ y en el segundo $(26,5 \%)$ (figura 2$)$.

\section{DISCUSIÓN}

Durante cinco temporadas, 2008-09 a 2012-13, el estudio cycEVA ha proporcionado estimaciones de EV antigripal estacional y pandémica en España en el marco de las redes de médicos y pediatras centinela integradas en el ScVGE. Durante la fase piloto $2008-09^{16}$ se demostró la viabilidad del estudio siendo una etapa crucial para afrontar el reto que supuso la estimación de la efectividad de la vacuna estacional 2009. 10 y la monovalente pandémica durante la pandemia de $2009^{18}$. Posteriormente el estudio cycEVA se consolidó a lo largo de las tres ediciones post-pandémicas ${ }^{19-22}$.

El estudio cycEVA se desarrolla en el marco del ScVGE, en el que los médicos y pediatras centinela de vigilancia de gripe sustentan una experiencia considerable en la toma de muestras y recogida de información a los pacientes que acuden a consulta. La aceptabilidad del estudio se evidenció en su fase piloto durante la temporada 2008$09^{16}$ y posteriormente se ha demostrado con el aumento del número de MP participantes a lo largo de las sucesivas ediciones. Ade- más se observa un incremento del número de MP que incluyen al menos un paciente en el periodo de estudio así como con una mejora en la recogida de información gracias a la continua adaptación del diseño de estudio para reducir la carga de trabajo de los médicos participantes (simplificación del cuestionario y mejora de sus controles de calidad).

La flexibilidad del estudio cycEVA ha permitido mejorar su eficiencia con la incorporación de sucesivos cambios al protocolo inicial: 1) la población de estudio se amplió ante la aparición del nuevo virus de la gripe $\mathrm{A}(\mathrm{H} 1 \mathrm{~N} 1) \mathrm{pdm} 09$ causante de la pandemia de 2009; 2) se seleccionó y consensuó el grupo control adecuado ${ }^{9}$ de forma que después de la fase piloto 2008-09 se adoptó un diseño de estudio test-negativo de casos y controles ${ }^{15}$ que en la actualidad está ampliamente aceptado por la comunidad científica $^{26-29}$; 3) se restringió la inclusión de pacientes al periodo de máxima circulación de virus gripales con el fin de evitar posibles sesgos metodológicos descritos previamente $^{11}$ y obtener una mejor relación caso/control'; y 4) se incorporaron al cuestionario nuevas variables de ajuste para controlar la ocurrencia de sesgos y confusión que pudieran estar invalidando metodológicamente los resultados ${ }^{7,30-33}$. A partir de la edición 2009-10 se recogió información sobre los dos nuevos factores de riesgo de complicaciones por gripe descritos en la pandemia de 2009, embarazo y obesidad mórbida (IMC $\geq 40 \mathrm{~kg} / \mathrm{m}^{2}$ ). Gracias a la incorporación de la variable "pertenencia a los grupos recomendados de vacunación", desde la edición 2010-11 se obtuvieron estimaciones de EV antigripal en España para los grupos diana de vacunación ${ }^{20-21}$. La inclusión de la variable "número de consultas al MP en los últimos 12 meses" permite ajustar las estimaciones de EV por la conducta de frecuentación médica (health seeking behavior) de los pacientes incluidos en el estudio ${ }^{28}$. 
Una mejor participación de los MP, la recogida de información de más calidad y la consolidación del diseño de estudio posibilitan la consecución de estimaciones más precisas y menos sesgadas en los distintos subgrupos analizados ${ }^{20-21}$. Una fortaleza de cycEVA es la mejora con el tiempo de su contribución al estudio europeo I-MOVE, convirtiéndose en un componente básico para obtener las estimaciones europeas. $\mathrm{La}$ disminución observada en la última edición consolidada 2012-13 con respecto a las anteriores se debió a la incorporación de un nuevo país en el estudio multicéntrico IMOVE que participó con el $43 \%$ del total de pacientes y el $39 \%$ de los casos confirmados en el estudio.

Cumpliendo con el objetivo de la red europea I-MOVE, el estudio cycEVA fue capaz de obtener estimaciones tempranas de EV en el pico de la onda epidémica desde la pandemia de $2009^{21,22}$. La disponibilidad de estas estimaciones tempranas permite, por una parte, contribuir a la toma de decisiones sobre estrategias de vacunación antigripal basadas en evidencias epidemiológicas sólidas, como es la puesta en marcha de medidas alternativas de prevención para los grupos de riesgo en escenarios con EV antigripal baja. Por otra, su contribución a la selección de las cepas a incluir en la vacuna antigripal. La oportunidad de las estimaciones de EV antigripal del estudio europeo I-MOVE, en el que España participa con cycEVA, hizo posible su contribución al informe GIVE 2013 (The Global Influenza Vaccine Effectiveness Collaboration $)^{34}$. GIVE surgió de un acuerdo de colaboración internacional para compartir las estimaciones de EV antigripal entre diferentes países de los hemisferios norte y sur, con el objetivo de apoyar a la OMS en el proceso anual de selección de cepas de virus gripales para la vacuna de la siguiente temporada, aportando datos epidemiológicos de EV antigripal. De esta forma, las estimaciones de EV antigripal, junto con resultados procedentes de la caracteriza- ción de virus y de estudios serológicos vacunales contribuyeron por primera vez en febrero de 2013, a la decisión del Comité de Selección de Cepas de la OMS sobre las recomendaciones de la composición de la vacuna antigripal en la próxima tempora$\mathrm{da}^{25,35}$.

La difusión de los resultados nacionales de EV antigripal se considera un pilar básico de todos los integrantes de la red IMOVE. A lo largo del período de estudio la difusión de resultados cycEVA se amplía con el uso de más formas de presentación (boletines semanales, informes técnicos semestrales y anuales, reuniones, congresos y publicaciones científicas) dirigidas a foros nacionales e internacionales: médicos participantes en el estudio, profesionales de salud pública y atención sanitaria, autoridades sanitarias, agencias de salud pública internacionales, y población general.

Con respecto al impacto de los resultados, cycEVA ha contribuido aportando evidencias científicas sobre el efecto protector de la vacunas antigripales. Además se han generado respuestas a interrogantes actuales relacionados con la inmunidad: efectividad de la vacunas pandémicas ${ }^{18}$, duración del efecto protector de la vacuna, influencia de la circulación de cepas discordantes ${ }^{20}$ y efecto de las vacunaciones antigripales repetidas temporada tras tempora$\mathrm{da}^{19}$.

Entre las limitaciones del estudio cycEVA se encuentra el insuficiente tamaño muestral conseguido que afecta a la precisión y fiabilidad de las estimaciones de EV, especialmente en los grupos de edad extremos, y para los análisis estratificados. Un mayor tamaño muestral se conseguiría aumentando el número de pacientes incluidos en el estudio por red participante $\mathrm{y} / \mathrm{o}$ aumentando el número de redes centinela participantes, lo que además mejoraría la representatividad territorial/nacional en las estimaciones de EV. 
Otras de las limitaciones del estudio es la baja cobertura vacunal de los pacientes incluidos, si bien la proporción de pacientes vacunados en personas mayores de 64 años concuerda con las estimaciones oficiales $^{4}$. Con respecto a los sesgos metodológicos del diseño test-negativo de casos y controles empleado cabe destacar que, aunque actualmente es el diseño más utilizado en los estudios observacionales sobre la efectividad de la vacuna antigripal, se siguen describiendo ciertos sesgos asociados con su uso ${ }^{4,10-14}$. Aunque se asume que los controles negativos y los casos presentan la misma conducta de frecuentación médica (health seeking behaviour), se sigue cuestionando si los controles reflejan adecuadamente la cobertura vacunal de la población que da origen a los casos.

Con el estudio cycEVA España participa en la red europea I-MOVE ${ }^{5}$, que se configura actualmente como una sólida plataforma para la monitorización de la efectividad de la vacuna antigripal en Europa. A pesar de ello, el principal desafío de cycEVA es su sostenibilidad. Si bien los estudios observacionales no son tan caros como los ensayos clínicos, necesitan un rigoroso control y coordinación para mantener la calidad, lo que hasta el momento eleva su coste por encima del de los actuales sistemas centinela de vigilancia de gripe.

La consolidación y la experiencia metodológica conseguida con el estudio cycEVA ha supuesto un valor añadido para el ScVGE, que se beneficia de un mayor rigor en la sistemática de toma de muestras y en la calidad de la información recogida. Por ello, cycEVA es una herramienta muy útil de validación y optimización de la información de vigilancia obtenida del ScVGE, con el objetivo de que en un futuro proporcione estimaciones anuales robustas de EV antigripal, que contribuyan a la prevención y control de la enfermedad.
La experiencia de estos cinco años de evaluación de la EV antigripal en España con el estudio cycEVA se traduce en un aumento en la precisión de las estimaciones y de la oportunidad en su difusión. Sus resultados contribuyen a orientar las políticas nacionales y europeas, como parte de la red I-MOVE, en relación a las estrategias anuales de vacunación antigripal. Por otra parte, cycEVA es una herramienta esencial de validación de la información obtenida en el ScVGE que facilita que este sistema de vigilancia sea capaz de asumir la vigilancia de la efectividad de la vacuna antigripal en España, guiando las medidas encaminadas al control de esta enfermedad.

\section{AGRADECIMIENTOS}

Agradecemos la excelente colaboración de los médicos y pediatras centinela participantes en el estudio cycEVA así como el apoyo de Marta Valenciano, Esther Kissling y Alain Moren, de Epiconcept.

\section{BIBLIOGRAFÍA}

1. SAGE working group. Background paper on influenza vaccines and immunization. Disponible en: http://www.who.int/immunization/sage/meetings/2012/april/1_Background_Paper_Mar26_v13_cl eaned.pdf?ua $=1$

2. Cox NJ, Subbarao K. Influenza. Lancet 1999;354: 1277-82. Disponible en: http://www.sciencedirect.com/science/article/pii/S014 0673699012416

3. Nicoll A, Ciancio B, Tsolova S, Blank P, Yilmaz C. The scientific basis for offering seasonal influenza immunisation to risk groups in Europe. Euro Surveill 2008;13: 1-8. Disponible en: http://www.eurosurveillance.org/images/dynamic/EE/V13N43/art19018.pdf

4. Ministerio de Sanidad, Servicios Sociales e Igualdad. Recomendaciones de vacunación antigripal. Disponible en: http://www.msssi.gob.es/profesionales/saludPublica/prevPromocion/vacunaciones/docs/Vacuna cionAntigripal.pdf

5. Influenza - Monitoring Vaccine Effectiveness. Disponible en: https://sites.google.com/site/epiflu/about-i-move 
6. Valenciano M, Ciancio B. I-MOVE: a European network to measure the effectiveness of influenza vaccines. Euro Surveill 2012; 17. Disponible en: http://www.eurosurveillance.org/images/dynamic/EE/V17N39/art20281. pdf

7. European Centre for Disease Prevention and Control. Protocol for case-control studies to measure pandemic and seasonal influenza vaccine effectiveness in the European Union and European Economic Area Member States. Disponible en: http://ecdc.europa.eu/en/publications/Publications/0907 TED Influenza AH1N1 Meas uring_Influenza_Vaccine_Effectiveness_Protocol_Case_Control_Studies.pdf

8. European Commission. European Commission. Commission Implementing Decision of 8 August 2012 amending Decision 2002/253/EC laying down case definitions for reporting communicable diseases to the Community network under Decision No 2119/98/EC of the European Parliament and of the Council, OJ L 2611/1, 27.9.12. Disponible en: http://eurlex.europa.eu/LexUriServ/LexUriServ.do?uri=OJ:L:201 2:262:0001:0057:EN:PDF

9. Valenciano M, Kissling E, Ciancio BC, Moren A. Study designs for timely estimation of influenza vaccine effectiveness using European sentinel practitioner networks. Vaccine 2010;28: 7381-8. Disponible en: http://www.sciencedirect.com/science/article/pii/S02644 $10 \mathrm{X} 10013186$

10. De SG, Skowronski DM, Wu XW, Ambrose CS. The test-negative design: validity, accuracy and precision of vaccine efficacy estimates compared to the gold standard of randomised placebo-controlled clinical trials. Euro Surveill 2013; 18. Disponible en: http://www.eurosurveillance.org/images/dynamic/EE/V18N37/art20585.pd f

11. Jackson ML, Nelson JC. The test-negative design for estimating influenza vaccine effectiveness. Vaccine 2013;31: 2165-8.

12. Foppa IM, Haber M, Ferdinands JM, Shay DK. The case test-negative design for studies of the effectiveness of seasonal influenza vaccine. Vaccine 2013; 3104-9. Disponible en: http://www.sciencedirect.com/science/article/pii/S0264410X13004659

13. Cowling BJ, Nishiura H. Virus interference and estimates of influenza vaccine effectiveness from test-negative studies. Epidemiology 2012;23: 930-1.

14. Sundaram ME, McClure DL, VanWormer JJ, Friedrich TC, Meece JK, Belongia EA. Influenza vaccination is not associated with detection of noninfluenza respiratory viruses in seasonal studies of influenza vaccine effectiveness. Clin Infect Dis 2013;57: 789-93.
15. Orenstein EW, De Serres G, Haber MJ, Shay DK, Bridges CB, Gargiullo P, et al. Methodologic issues regarding the use of three observational study designs to assess influenza vaccine effectiveness. Int J Epidemiol 2007;36: 623-31. Disponible en: http://ije.oxfordjournals.org/content/36/3/623.full.pdf + html

16. Savulescu C, Valenciano M, de Mateo S, Larrauri A, the cycEVA Study Team. Estimating the influenza vaccine effectiveness in elderly on a yearly basis using the Spanish influenza surveillance network--pilot case-control studies using different control groups, 2008-2009 season, Spain. Vaccine 2010;28: 2903-7.

17. Agresti A. An introduction to categorical data analysis. Second edition ed. Hoboken, New Jersey: JohnWiley \& Sons; 2007.

18. Savulescu C, Jimenez-Jorge S, De Mateo S et al. Using surveillance data to estimate pandemic vaccine effectiveness against laboratory confirmed influenza A(H1N1)2009 infection: two case controls studies, Spain, season 2009-2010. BMC Public Health 2011;11: 899-907.

19. Jimenez-Jorge S, Savulescu C, Pozo F, de MS, Casas I, Ledesma J, et al. Effectiveness of the 2010-11 seasonal trivalent influenza vaccine in Spain: cycEVA study. Vaccine 2012;30: 3595-602.

20. Jimenez-Jorge S, de MS, Delgado-Sanz C, Pozo F, Casas I, Garcia-Cenoz M, et al. Effectiveness of influenza vaccine against laboratory-confirmed influen$\mathrm{za}$, in the late 2011-2012 season in Spain, among population targeted for vaccination. BMC Infect Dis 2013;13: 441. Disponible en: http://www.biomedcentral.com/content/pdf/1471-2334-13-441.pdf

21. Savulescu C, Jimenez-Jorge S, de Mateo S, Ledesma J, Pozo F, Casas I, et al. Effectiveness of the 2010/11 seasonal trivalent influenza vaccine in Spain: preliminary results of a case-control study. Euro Surveill 2011;16: 1-6. Disponible en: http:/www.eurosurveillance.org/images/dynamic/EE/V16N11/art19820. pdf

22. Jimenez-Jorge S, de MS, Pozo F, Casas I, Garcia $\mathrm{CM}$, Castilla J, et al. Early estimates of the effectiveness of the 2011/12 influenza vaccine in the population targeted for vaccination in Spain, 25 December 2011 to 19 February 2012. Euro Surveill 2012;17: 1-6. Disponible en: http:/www.eurosurveillance.org/images/dynamic/EE/V17N12/art20129.pdf

23. Osterholm MT, Kelley NS, Sommer A, Belongia EA. Efficacy and effectiveness of influenza vaccines: a systematic review and meta-analysis. Lancet Infect Dis 2011;12: 36-44. Disponible en: http://www.sciencedirect.com/science/article/pii/S147330991170295X 
24. Girard MP, Katz JM, Pervikov Y, Hombach J, Tam JS Report of the 7th meeting on Evaluation of Pandemic Influenza Vaccines in Clinical Trials, World Health Organization, Geneva, 17-18 February 2011. Vaccine 2011;29: 757986.

25. World Health Organizazion. Weekly epidemiological record. Review of the 2011-2012 winter influenza season, northern hemisphere. 2012; 87. p.233. Disponible en: http://www.who.int/wer/2012/wer8724.pdf

26. Kissling E, Valenciano M, Larrauri A, Oroszi B, Cohen $\mathrm{J}$, Nunes B, et al. Low and decreasing vaccine effectiveness against influenza A(H3) in 2011/12 among vaccination target groups in Europe: results from the I-MOVE multicentre case-control study. Euro Surveill 2013; 18. Disponible en: http://www.eurosurveillance.org/images/dynamic/EE/V18 N05/art20390.pdf

27. Fielding JE, Grant KA, Tran T, Kelly HA. Moderate influenza vaccine effectiveness in Victoria, Australia, 2011. Euro Surveill 2012;17: 1-5. Disponible en: http://www.eurosurveillance.org/images/dynamic/EE/V17N11/art20115.p df

28. Belongia EA, Kieke BA, Donahue JG, Coleman LA, Irving SA, Meece JK, et al. Influenza vaccine effectiveness in Wisconsin during the 2007-08 season: Comparison of interim and final results. Vaccine 2011;29: 6558-63. Disponible

en:

http://www.sciencedirect.com/science/article/pii/S0264410 X11010231

29. Skowronski DM, Masaro C, Kwindt TL, Mak A, Petric $\mathrm{M}, \mathrm{Li}$ Y, et al. Estimating vaccine effectiveness against laboratory-confirmed influenza using a sentinel physician network: results from the 2005-2006 season of dual A and B vaccine mismatch in Canada. Vaccine 2007;25: 2842-51. Disponible en: http://www.sciencedirect.com/science/article/pii/S0264410X06011054

30. Kenneth J.Rothman, editors.Epidemiología Moderna. Ediciones Díaz de Santos S.A ed. 1987; 42, Diseños de estudios epidemiológicos. p. 144-54.

31. Hak E, Verheij TJ, Grobbee DE, Nichol KL, Hoes AW. Confounding by indication in non-experimental evaluation of vaccine effectiveness: the example of prevention of influenza complications. J Epidemiol Community Health 2002;56: 951-5. Disponible en: http://www.ncbi.nlm.nih.gov/pmc/articles/PMC1756997/p df/v056p00951.pdf

32. Valenciano M, Ciancio B, Moren A. First steps in the design of a system to monitor vaccine effectiveness during seasonal and pandemic influenza in EU/EEA Member States. Euro Surveill 2008;13: 1-8. Disponible en: http://www.eurosurveillance.org/images/dynamic/EE/V13 N43/art19015.pdf
33. Jackson LA, Jackson ML, Nelson JC et al. Evidence of bias in estimates of influenza vaccine effectiveness in seniors. Int J Epidemiol 2006;35: 337-44.

34. European Centre for Disease Control and Prevention (ECDC). ECDC Reviews: WHO Recommendation on influenza virus vaccines for the Northern $\mathrm{He}$ misphere 2013-2014 season. Disponible en: http://www.ecdc.europa.eu/en/activities/sciadvice/ layouts/forms/Review DispForm.aspx?ID=737\&List=a 3216f4c-f040-4f51-9f77-a96046dbfd72

35. World Health Organization. Recommended composition of influenza virus vaccines for use in the 2012-2013 northern hemisphere influenza season. Disponible en: http:/www.who.int/influenza/vaccines/virus/recommendations/201202_recommendation.pdf 